\title{
Planning the Protection and Digital Construction of Mount Tai Stone Inscriptions
}

\author{
Liang Mingying ${ }^{1, *}$, Cheng Xuening ${ }^{2}$ \\ ${ }^{1}$ Tourism, Resources, and Environment Laboratory, Taishan University, Taian, Shandong \\ ${ }^{2}$ Smart City Department, Zhengyuan Geomatics Group Co., Ltd. Shandong Branch, Jinan, Shandong
}

\author{
ARTICLE INFO \\ Article History \\ Received 03 November 2020 \\ Accepted 19 February 2021 \\ Keywords \\ Mount Tai stone inscriptions \\ cultural heritage \\ spatial information technology \\ digital protection
}

\begin{abstract}
After thousands of years of exposure to the elements, the Mount Tai stone inscriptions have been damaged to varying degrees. As immovable cultural relics, they are inseparable from their surrounding environment, and therefore continue to face numerous risks and safety hazards. Several spatial information technologies are used in this paper to perform tasks related to the Mount Tai stone inscriptions, including digital information acquisition, spatial database construction, and 3D visualization information system design. These functions support digital data storage, information-based management; 3D visual display of the Mount Tai stone inscriptions; remote monitoring is performed using intelligent networked devices; innovate digital communication and heritage culture transmission models of heritage culture; build a digital heritage protection system for the Mount Tai stone inscriptions. Based on principles of digital construction, this paper further explores methods for planning risk assessment and protective measures for the Mount Tai stone inscriptions, as well as for improving the digital archiving and modeling of the stone inscriptions and the sustainable development of cultural tourism products.
\end{abstract}

(C) 2021 The Authors. Published by Atlantis Press B.V This is an open access article distributed under the CC BY-NC 4.0 license (http://creativecommons.org/licenses/by-nc/4.0/).

\section{INTRODUCTION}

The protection of cultural heritage has been a topic of major interest to countries around the world and the institutions they charge with safeguarding their history. With the advent of the Information Age, the use of digital models to reconstruct and archive cultural artifacts is an important means for protecting the veracity and integrity of these cultural heritages, and ensuring that they can be displayed and protected effectively [1-3]. Mount Tai, which is located in the central part of China's Shandong Province, was one of the world's first listed natural and cultural heritages, and one of its most ancient places of religious and cultural significance (Figure 1). The cliff stone carvings of Mount Tai are its most important and distinctive cultural heritages, and are located across the official scenic area of Mount Tai, covering a region of more than $140 \mathrm{~km}^{2}$. These stone carvings are exposed to the natural environment, and as such have been damaged to varying degrees by many factors, including solar radiation and erosion from rain, wind, and sand. In 2017, the "Research and Demonstration of the Digital Protection Standard System of Cultural Relics and Essential Criteria", a Chinese national science and technology project initiated during the country's 12th Five-Year Plan, passed its initial inspection and began providing a standard and basis for the digital imaging and reconstruction of cultural heritage artifacts and relics. The theory and practice of the digital reconstruction of cultural heritage provides theoretical support and reference value for studying the digital protection of cultural heritage [4,5]. Digitization has been widely applied to the protection of cultural relics, partly resulting from the fact that issues of space management and safety monitoring

Corresponding author. Email: liangmingying@tsu.edu.cn of immovable cultural relics remain difficult problems that have yet to be solved. 3D laser scanning technology can be used to maintain the status quo of cultural relics exactly, authentically, and completely, providing true and detailed scientific data for future maintenance, restoration, and academic research work [6-9]. This technology has been applied to the construction and protection of records regarding immovable cultural relics. The first local Chinese standards for the digitization of stone cultural relics were the Technical Regulations for the Digital Acquisition of the Cultural Relics in Cave Temples via 3D Laser Scanning and the Technical Regulations for 3D Digital Acquisition of Cultural Relics in Cave Temples for Close-range Photogrammetry [10], which were released by Shanxi Province in 2019. At present, the number of digital studies on the Mount Tai stone inscriptions remains few. In 2009, Liu and Wang [11] used 3Dmax software to perform research and practice of 3D modeling of individual Mount Tai stone inscriptions, but the overall digital construction of Mount Tai stone inscriptions was not started yet. In the context of China's national big data cultural system construction, it is of great significance to learn to comprehensively utilize multiple spatial information technologies to study issues of digitally protecting and managing the Mount Tai stone inscriptions, to expand methods of heritage protection, and innovate cultural communication modes to build a digital protection system for the Mount Tai stone inscriptions.

\section{RISKS AND PROTECTION STATUS OF THE MOUNT TAI STONE INSCRIPTIONS}

Most of the Mount Tai stone inscriptions are cliff carvings engraved on complex rocks (i.e., metamorphic rocks formed by migmatization 


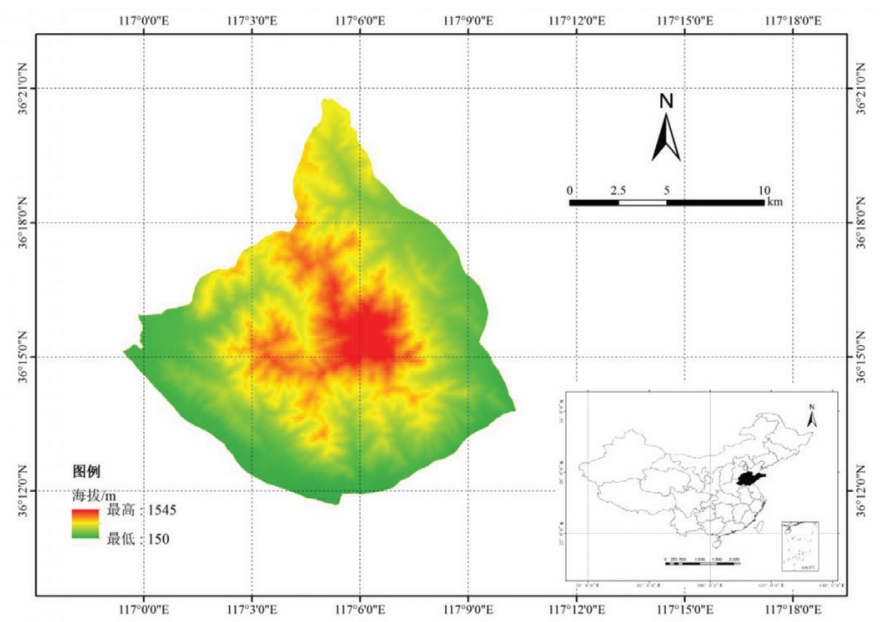

Figure 1 Terrain map of Mount Tai.

during the Archaeozoic era). This type of rock is of high density and hardness and is resistant to weather, but as an immovable cultural relic, they may not remain well-preserved forever. Due to the inseparability of the stone inscriptions from the environment, the protection of Mount Tai stone inscriptions can be subdivided into questions of protecting the stone inscriptions themselves as well as the environment surrounding them (Figure 2). To protect the Mount Tai stone inscriptions, questions of the importance, sensitivity, and threats to the stone inscriptions and the environment should be taken into comprehensive consideration.

\subsection{Protection of Stone Inscriptions}

The stone inscriptions are considered to be cultural relics, but their assessment as cultural relics includes multiple standards and variables. Other main indicators include age, creator, content, and the value of the calligraphy used, as well as their sensitivity and degree of threat. Potential threats include corrosion, weathering, cracking, cleavage, collapse, falling rocks, artificial destruction, sun exposure, temperature differentials, rainwater, vegetation, and an increase in the number of tourists [12,13] (Figure 2). The Sutra Rock Valley in Mount Tai, a key cultural relic site under official state protection, suffers from multiple types of damage, including cracks, fractures, surface exfoliation, biological damage, salt precipitation, and rust crusting, and these are caused by many different factors [14].

At present, the Mount Tai stone inscriptions are primarily protected by physical and chemical means. For example, all of the "Inscription to Honor Mount Tai by Tang Xuanzong" is protected by gilding; fences and footpaths are built beside the stone inscriptions near touring trails to isolate tourists from the stone inscriptions and guide their visits; in areas with hidden geological risks such as faults, after geological surveys have been performed, massifs subject to collapse or fracture were reinforced by grouting. The stone inscriptions that have been damaged to some extent are checked on and maintained regularly, for example with the use of anti-weathering paint to trace black ink over characters printed in red every 3 years, or other forms of chemical protection. Monitoring facilities are mounted near key cultural relics to perform timely identification of various safety hazards so that protective measures can be taken rapidly. Physical protection tends to function well, but lasting
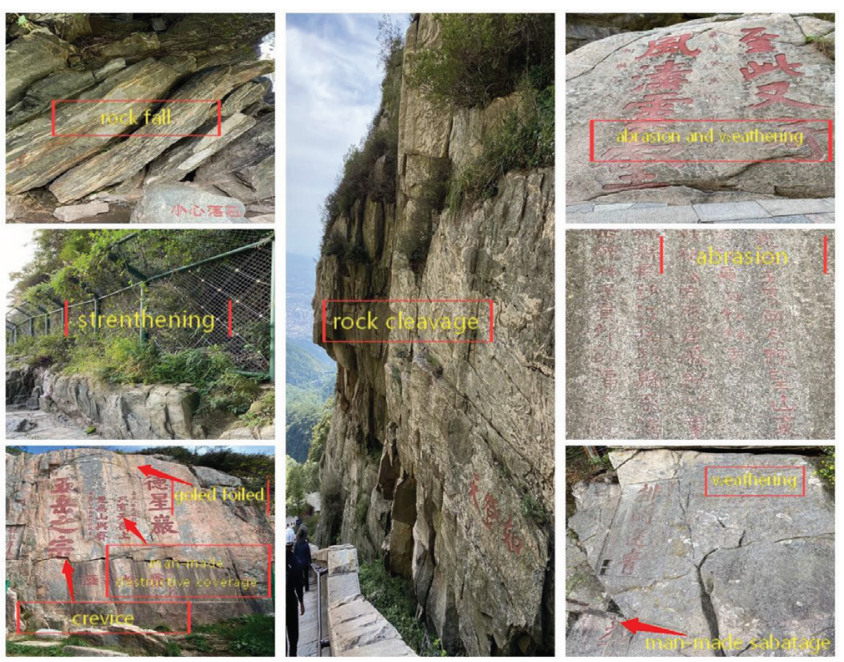

Figure 2 Risks in protecting the Mount Tai stone inscriptions.

positive or negative effects of chemical paints on stone wear may not appear over short periods of time, so long-term monitoring and research are required.

\subsection{Protection of the Environment}

The historical and natural landscape of the environment surrounding the stone inscriptions is an important component of stone inscription valuation systems. The protection of stone inscriptions should be implemented after a comprehensive assessment of the degree of sensitivity and threat faced by the surrounding environment. For example, Sutra Rock Valley were originally engraved on a boulder in a tranquil valley surrounded by hills. In 1967, a dam was built upstream of the valley, resulting in the local waterway being moved to the west side of the carved stone. The nearby Mountain and Flowing Water Pavilion was moved from its former site to the current location. Later, stone railings and ladders were built around the boulder to prevent visiting tourists from approaching too closely and making rubbings, thus protecting the stone inscriptions against water erosion and tourist activities. The numerous cliff carvings on Mount Tai were an important part of cultural tourism experiences on Mount Tai. For a long time, tourist activities have had a certain impact on the surrounding ecological environment. The greater the impact of human activities on the stone inscriptions, the greater the threat posed to the stone inscriptions. Therefore, environmental safety monitoring and protection is also an important part of the protection of immovable cultural relics.

\section{PLANNING THE DIGITAL CONSTRUCTION OF MOUNT TAI STONE INSCRIPTIONS}

\subsection{Design of Mount Tai Stone Inscription Information System based on 3D GIS}

The Mount Tai stone inscriptions are immovable cultural relics, and are characterized by their high number and wide distribution. A Geographic Information System (GIS) can be used as a data 
management and system development platform to construct a 3D visualization information system for the Mount Tai stone inscriptions. This can help to solve problems concerning the Mount Tai stone inscriptions, including their digital protection, spatial management, comprehensive search processes, real-time monitoring, safety warnings, and more. The architecture of this system is shown in Figure 3. It is divided into five layers, from the bottom up, including a perception layer, data layer, engine layer, service layer, and application layer, including comprehensive searching, 3D browsing, and space management of stone inscriptions. Also, intelligent environmental monitoring equipment can be used to provide continuous real-time monitoring of environmental temperature and humidity, as well as providing alarms in response to intrusion.

\subsection{Construction Method}

\subsubsection{Digital information acquisition}

Information acquisition processes are divided into two parts: the acquisition and processing of basic geographic data, and the acquisition and processing of the stone inscription data. The digital construction of the Mount Tai stone inscriptions needs to be carried out on the basis of the geographic information of Mount Tai. This geographical data includes the imaging data of the Mount Tai Scenic Area (Administrative Area) and local topographic DEM data. The sample stone inscription data is partitioned on the basis of

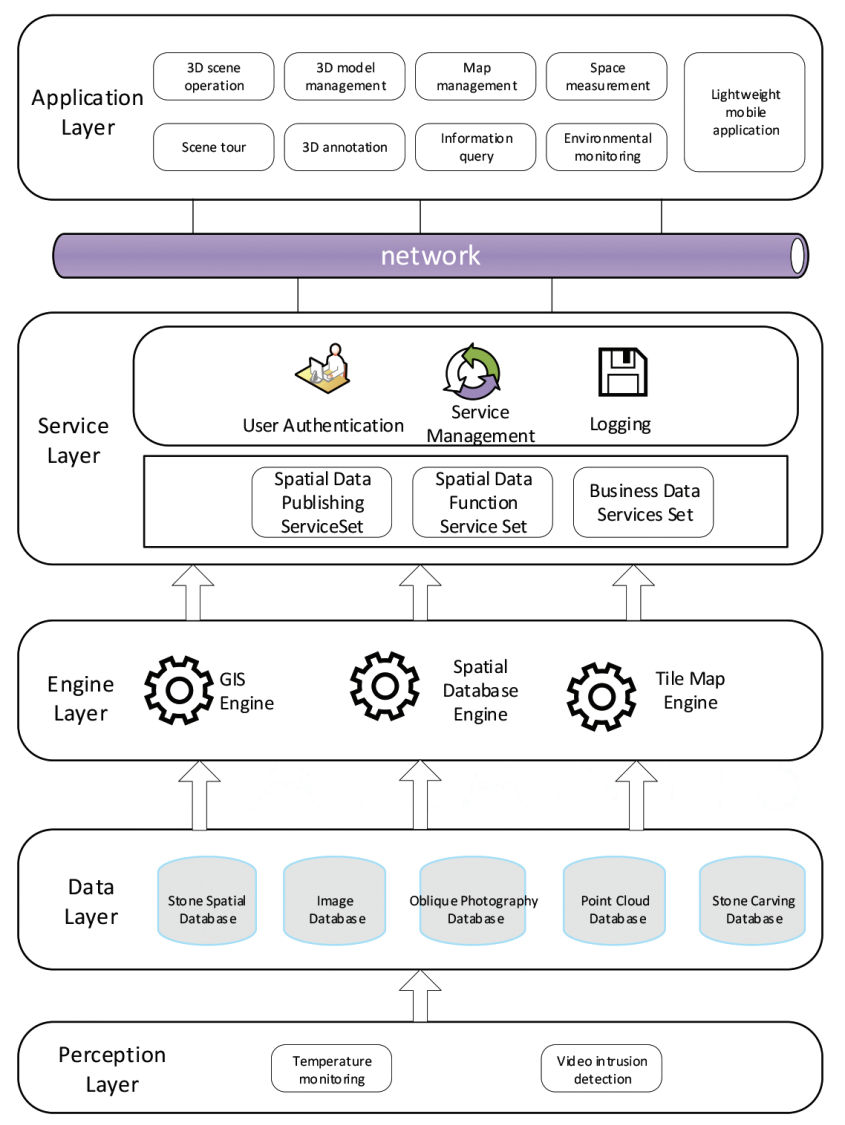

Figure 3 Architecture of 3D Mount Tai Stone inscription visualization information system. the records of the Mount Tai stone inscriptions [15] and are justified by this basic information. Priority is given to the stone inscriptions between the Red Gate of Taishan Middle Road to the summit of Mount Tai, while four key cultural relics protection units are designated as key areas: the Sutra Rock Valley, the Yunbu Bridge, the Yuhuang Peak, and the Daguan Peak.

\section{(1) Processing of basic geographical data}

The basic geographic data are used as the basis for the digitization of the Mount Tai stone inscriptions, providing a truly 3D geographic spatial framework. Publicly available topographical and image servicing data are used to construct a $3 \mathrm{D}$ display scene for the Mount Tai stone inscriptions, while the topographic DEM data provide a terrain mesh model for the Mount Tai Scenic Area, and the image data is used to enhance the realism of the macrogeographical environment. The data range covers an area of about $140 \mathrm{~km}^{2}$, and this is equal to the administrative range of the Mount Tai Scenic Area.

(2) Digital information acquisition and processing of Mount Tai stone inscriptions

The acquired digital information includes the images, content and the attributes of the stone inscriptions, and their spatial environment.

The information of the stone inscriptions and their spatial environment is collected on the principle of minimal interference with cultural relics. Considering the complexity of the mountain environment, contact-free measurement methods (panoramic camera technology, oblique photography technology, and laser point cloud technology) are used to keep a full record of the geometric information of the stone inscriptions, as well as their texture and surrounding environment, and these cover multiple spatial scales. The panoramic camera technology used is an HD SLR camera used to take $360^{\circ}$ panoramic photos of the Mount Tai stone inscriptions; oblique photography technology is mainly used to acquire the spatial location and texture information of the stone inscriptions, i.e., to create high-precision records and 3D modeling of key individual stone inscriptions and the spatial information of the calligraphy to complete the acquisition of related information in a multidimensional space, including data collected different spatial scales and in different environments (Figure 4) (Top: a 3D model of the Sutra Rock Valley made using oblique photogrammetry; Bottom: an individual character model by 3D printing based on laser point cloud data.).

The attribute information of Mount Tai stone inscriptions is based on the official records of the Mount Tai stone inscriptions [15], which provide the main reference data and fundamental basis for the related database. Based on other research literature on the Mount Tai stone inscriptions, the information about the current state of the stone inscriptions is aggregated and the data are standardized. To digitize of the attribute information of the Mount Tai stone inscriptions, a detailed data sheet is prepared through both literature retrieval and on-the-spot investigation. A thematic database has also been designed for the Mount Tai stone inscriptions. During the field survey, we collected the information about the geographic location of the stone inscriptions, took HD photos, and then verified the location, shape, specification, and integrity of the stone inscriptions one-by-one to provide basic data for the establishment of a 3D model of the stone inscriptions. 


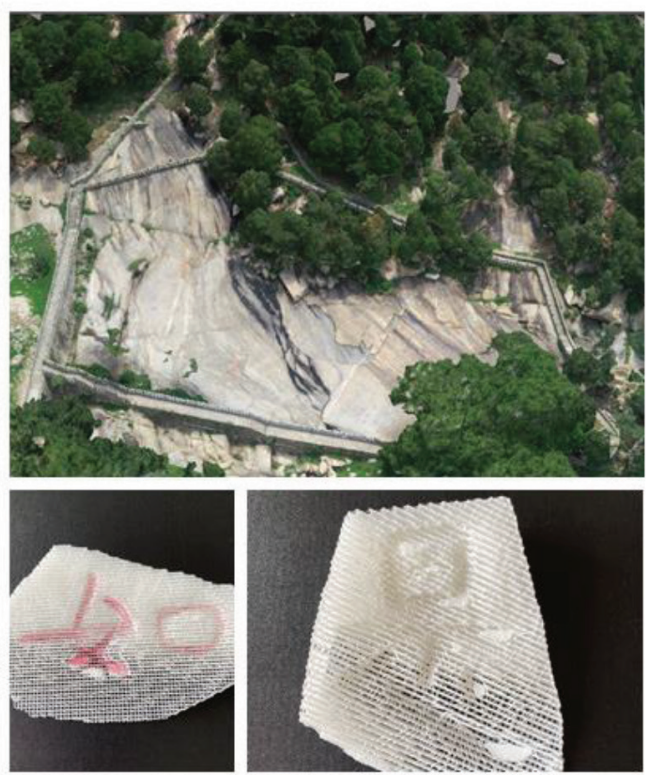

Figure 4 VR model of Sutra Rock Valley (local).

\subsubsection{Database construction}

The database of Mount Tai stone inscriptions is made up of a spatial database and a thematic database. The spatial database of the Mount Tai stone inscriptions mainly involves the stone inscriptions' oblique photogrammetry data, point cloud data, and spatial data. After the acquisition of the oblique photogrammetry data, professional software was used to process and export it in OSGB format. The point cloud data was processed and exported in LAS or XYZ format. The spatial data of the stone inscriptions was processed on the GIS platform. After being processed on the GIS platform, the spatial data was uniformly released as map data to support multi-terminal data display. The thematic database of the stone inscriptions aims to digitize the acquired and collated textual information of the Mount Tai stone inscriptions and, after classification, import it into the database to establish a static file catalog for the pictures and panoramic image data, thereby facilitating the development of subsequent applications and services (Table 1).

\subsubsection{Functional design}

The $3 \mathrm{D}$ visualization information system of the Mount Tai stone inscriptions consists of four functional modules, including intelligent monitoring, 3D display, stone inscription management, and cultural tourism modules (Figure 5).

(1) Intelligent monitoring. As immovable cultural relics, the Mount Tai stone inscriptions are displayed in a completely open natural space and cannot be protected in a closed state. Remote intelligent terminal sensing equipment and video monitoring equipment are used to perform real-time monitoring of key stone inscriptions, and any stone inscriptions in severe danger or with hostile environments are handled in accordance with requirements for cultural relic protection. An early warning is issued whenever a risk is identified. The risk is then displayed via integrated video within the $3 \mathrm{D}$
Table 1 Mount Tai stone inscription data composition

\begin{tabular}{|c|c|c|}
\hline Database & Data type & Description \\
\hline \multirow[t]{3}{*}{$\begin{array}{l}\text { Spatial } \\
\text { database }\end{array}$} & Oblique photograph data & $\begin{array}{l}\text { Records the actual 3D data of the } \\
\text { Mount Tai stone inscriptions } \\
\text { and the environment. }\end{array}$ \\
\hline & Point cloud data & $\begin{array}{l}\text { Records high-precision 3D } \\
\text { data about the key Mount Tai } \\
\text { stone inscriptions, such as } \\
\text { font shape, concave-convex } \\
\text { degree, etc. }\end{array}$ \\
\hline & $\begin{array}{l}\text { Spatial database of } \\
\text { stone inscriptions }\end{array}$ & $\begin{array}{l}\text { Records the 3D coordinate } \\
\text { Information of the Mount Tai } \\
\text { stone inscriptions, including } \\
\text { the longitude, latitude, } \\
\text { altitude, orientation, etc. }\end{array}$ \\
\hline \multirow[t]{3}{*}{$\begin{array}{l}\text { Thematic } \\
\text { database }\end{array}$} & Image data & $\begin{array}{l}\text { Records information about the } \\
\text { stone inscriptions and the } \\
\text { actual environment (can be } \\
\text { collected seasonally). }\end{array}$ \\
\hline & $\begin{array}{l}\text { Current state data of } \\
\text { stone inscriptions }\end{array}$ & $\begin{array}{l}\text { Records the name, age, author, } \\
\text { content, font and number of } \\
\text { the extant stone inscriptions. }\end{array}$ \\
\hline & $\begin{array}{l}\text { Literature data of stone } \\
\text { inscriptions }\end{array}$ & $\begin{array}{l}\text { Records literature related to the } \\
\text { stone inscriptions. }\end{array}$ \\
\hline
\end{tabular}

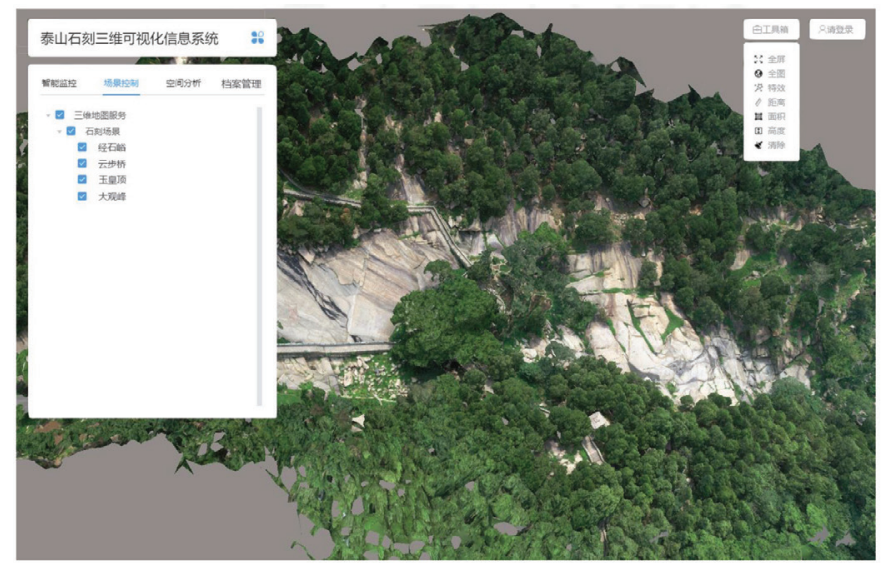

Figure 5 Screenshot of Mount Tai stone inscriptions information system.

visualization system to enhance the level of on-site surveillance perception.

(2) 3D display. The system supports the viewing of the 3D Mount Tai stone inscription model data based on their geospatial location. These viewing methods include $360^{\circ}$ panoramic images, an oblique photography model, and a point cloud model. Users can choose different 3D scenes for their browsing. In addition, route roaming can be adopted for browsing within the 3D space of Mount Tai. Spatial measurement and 3D interest point labeling can also be performed.

(3) Stone inscription search queries and record management. Ordinary users can query the basic information, spatial information, pictures, videos, and 3D model information of stone inscriptions through the platform's search function. After login, the platform administrator can edit, modify, add, and delete stone inscriptions data spreadsheets. 
(4) Cultural tourism. To support both tourists and the public, the Mount Tai stone inscriptions are displayed in a lightweight app to help promote Mount Tai's stone inscriptions through publicly accessible $360^{\circ}$ panoramic images, voice recordings, and texts.

\section{CONCLUSION AND DISCUSSION}

\subsection{Conclusion}

Based on 3D GIS platform technology, a spatial database is constructed for the Mount Tai stone inscriptions by building a 3D visualization information system which digitally acquires and processes the basic geographic information of Mount Tai. The spatial information and attribute information of the stone inscriptions is used to realistically display the current state of the Mount Tai stone inscriptions, providing spatial visualization management of the cultural relics as well as a stone inscription information search function, record maintenance and 3D spatial operation, real-time monitoring of particularly important, highly sensitive, and highly threatened stone inscriptions and environmental spaces containing potential damage and risks. Protective measures are taken in rapid response to provide preventive protection. Based on $3 \mathrm{D}$ visualization processes, it is possible to further design a highly narrative Mount Tai cultural display project that boasts immersive experiences and allows visitors to deeply integrate themselves in the historical features and cultural meanings of the Mount Tai stone inscriptions so as to promote the transmission and awareness of this ancient heritage culture.

\subsection{Discussion}

Digitization has enabled the authentic recording and preservation of the cultural heritage, but the Mount Tai stone inscriptions remain at the risk of suffering weathering, corrosion, cleavage, collapse, ecological changes, geological disasters, and artificial destruction, all of which pose a threat to the permanent preservation of the stone inscriptions. However, which preventive measures can be taken to minimize the damage caused to the stone inscriptions by these factors?

This paper proposed the use of the following three preventive measures: First, risk monitoring and early warning. A real-time monitoring and early warning system can be continuously constructed for the Mount Tai stone inscriptions based on their 3D visualization information system, thus providing real-time monitoring and recording of key stone inscriptions, stone inscriptions under major threat, and various factors in their spatial environments, including the status of the stone inscriptions, 3D text \& calligraphy data, tracking of meteorological elements and ecological elements, tracking the number and behavior of tourists, observations of geological phenomena, etc. A risk threshold can be set in accordance with the relevant regulations on cultural relic protection so that early warnings can be given on a timely basis. The second aspect of the protection system is risk assessment. The risks faced by the stone inscriptions can be assessed by category and grade, while pertinent preventive measures can be developed as well. The third plank is composed of analysis of the stone inscriptions and environmental data, providing long-term monitoring. The causes of any risks and damage can be studied to provide experimental research-based formulation of protective measures to take advance precautions.

The database of the stone inscriptions can be further improved based on current and ongoing research to keep a comprehensive digital record of the Mount Tai stone inscriptions. At present, there is a limited amount of sample data in the stone inscriptions' visualization information system, and some data has yet to be included. Second, the stone inscriptions database is currently only able to collect, collate, and store presently available stone inscription data, image information, spatial information, and 3D data, and historical data on the stone inscriptions has not been entered into the database.

To resolve these issues, the Mount Tai stone inscriptions data entries must be updated and completed as soon as possible. Second, the database can be associated or jointly integrated with other stone inscription literature databases to expedite the digital recording of the Mount Tai stone inscriptions.

In addition, developing cultural tourism products through digital means in the context of culture-tourism fusion is also an important means to improve stone inscriptions culture protection systems, and thereby support cultural transmission and protection. Based on the unique spatial management and spatial analysis functions of the GIS platform, thematic tourism can be designed on the basis of the Mount Tai stone inscription culture to focus on promoting Mount Tai stone inscription touring pathways, including search queries of scenic spot information, interpretation of stone inscription culture and art, and other functions. A stone inscription-themed guide map can also be designed, including audio tours, panoramic views, and reading of stone inscription literature. These functions help the public acquire an in-depth knowledge of stone inscription culture, study the stone inscriptions, and exchange ideas on the stone inscriptions. On the one hand, tourists can be brought to the attention of the Mount Tai stone inscriptions and Mount Tai culture to strengthen their interest in traveling to Mount Tai. On the other hand, it will be possible to make up for any tourists' failure to observe Mount Tai's stone inscriptions and inform themselves of the deeper cultural context during their travels to Mount Tai, and thereby meet the demand for deeper understandings of Mount Tai culture.

\section{CONFLICTS OF INTEREST}

The authors declare they have no conflicts of interest.

\section{REFERENCES}

[1] Sun H. Study of cultural heritage and preserving cultural heritage-focusing on the categorization of cultural heritage studies. Stud Nat Culture Heritage 2018;3:1-9.

[2] Cao BW. Noumenon, information, value, function: some theoretical questions about the protection and inheritance of cultural heritage. China Culture Heritage 2019;48-53.

[3] Zhao T, Tao XW. The enlightenment of Japan's digitalization of cultural heritage. J Stud Culture Art Commun 2018;11:19-27.

[4] Liu P. Visual reconstruction-digital reconstruction of cultural heritage. Beijing, China: Tsinghua University Press; 2016. 
[5] China Economic Net. Home Page, The Current Politics Comprehensive, Culture, Text. Available from: http://www. ce.cn/2012sy/szzh/wh/202005/26/t20200526_34976567.shtml.

[6] Tang YZ, Huang M. Digital reconstruction of cliff stone using three-dimensional laser scanning measurement systems. Geomat Spat Inform Technol 2015;38:168-70.

[7] Sun BY, Weng YS, Zhou XJ. Application of reproduction-based 3D modeling technology to digitally reconstruct the cliff carvings. Sci Conserv Archaeol 2018;30:110-14.

[8] Xu YP. Research on the application of spatial information technology in the field of cultural relic protection. China Cult Heritage 2017;26-31.

[9] Ge HD, Deng SY. A research on 3-D digitalization protection and virtual recovery of tomb stone sculptures of southern dynasties. Urban Geotech Invest Survey 2014;14-16.
[10] The first local industry standard of Shanxi cultural museum systems released and implemented. Popular Standardization 2019;84.

[11] Liu ZJ, Wang CH. Three-dimensional real visualization of the Mount Tai stone carvings. Inform Technol 2009;591-3.

[12] Zhang BK. Research on the integral conservation and exhibition of cliff carvings with settings in the Mount Tai mountain world heritage site. Beijing, China: Tsinghua University; 2016.

[13] Liu Q. Protection of stone cultural relics. Beijing, China: Science Press; 2012.

[14] Zong SQ, Shi DY, Ming H, Chen Z, Liu P. Research on the causes of defects in stone valley carvings and the detection of stone cultural relics in Mountain Tai. Res Grotto Temples 2019;307-28.

[15] Yuan MY. Mount Tai stone inscriptions. Beijing, China: Zhonghua Book Company; 2007. 